Pacific Journal of Mathematics

TWO REMARKS ON ELEMENTARY EMBEDDINGS OF THE THOOAS J. TeCH 


\title{
TWO REMARKS ON ELEMENTARY EMBEDDINGS OF THE UNIVERSE
}

\author{
Thomas J. JECH
}

The paper contains the following two observations: 1 . The existence of the least submodel which admits a given elementary embedding $j$ of the universe. 2. A necessary and sufficient condition on a complete Boolean algebra $B$ that the Cohen extension $V^{B}$ admits $j$.

A function $j$ defined on the universe $V$ is an elementary embedding of the universe if there is a submodel $M$ such that for any formula $\varphi$,

$$
\forall x_{1}, \cdots, x_{n}\left[\varphi\left(x_{1}, \cdots, x_{n}\right) \longleftrightarrow M \vDash \varphi\left(j x_{1}, \cdots, j x_{n}\right)\right] .
$$

Let $j$ be an elementary embedding of the universe. If $N$ is a submodel, let $j_{N}=j \mid N$ be the restriction of $j$ to $N . N$ admits $j$ if (**) $\quad N \vDash j_{N}$ is an elementary embedding of the universe.

If $B$ is a complete Boolean algebra, let $V^{B}$ be the Cohen extension of $V$ by $B$. $V^{B}$ admits $j$ if

$\left({ }^{* *}\right) \quad V^{B} \vDash$ there exists an elementary embedding $i$ of the universe such that $i \supseteqq j$

THEOREM 1. There is a submodel $L(j)$ which is the least submodel which admits $j .^{1}$

Theorem 2. The Cohen extension $V^{B}$ admits $j$ if and only if the identity mapping on $j^{\prime \prime} B$ can be extended to a $j(V)$-complete homomorphism of $j(B)$ onto $j^{\prime \prime} B$.

Before giving the proof, we have a few remarks. The underlying set theory is the axiomatic theory $B G$ of sets and classes of Bernays and Gödel [1]. The formula $\varphi$ in $\left(^{*}\right)$ is supposed to have only set variables. However, if for any class $C$ we let $j(C)=\cup_{\alpha \in O_{n}} j\left(C \cap V_{\alpha}\right)$, then $\left(^{*}\right)$ holds also for formulas having free class variables ("normal formulas" of [1].) Incidentally, " $j$ is an elementary embedding of the universe" is expressible in the language of $B G$ (viz.: $j$ is an $\varepsilon$-isomorphism and $\forall C_{1} \forall C_{2}\left[\mathscr{F}_{i}\left(j C_{1}, j C_{2}\right)=j\left(\mathscr{F}_{i}\left(C_{1}, C_{2}\right)\right)\right]$ where $\mathscr{F}_{i}$ are the Gödel operations).

1 This was observed independently by K. Hrbáček, giving a different proof. 
A submodel $M$ is a transitive class containing all ordinals which is a model of $G B$; the classes of $M$ are all those subclasses $C$ of $M$ which satisfy the condition $\forall \alpha\left(C \cap V_{\alpha} \in M\right)$. The submodel $M$ in (*) is unique and $M=j(V)$. It is a known fact that if $j$ is not the identity then there exists a measurable cardinal. And, as proved recently by Kunen [2], $j(V) \neq V$. On the other hand, if there exists a measurable cardinal, then there exists a nontrivial elementary of the universe (cf. Scott [6]).

The notion $L(j)$ differs somewhat from the notion of relative constructibility, introduced by Lévy [4]; in general, $L(j) \supseteqq L[j]$.

A homomorphism is $C$-complete, if it preserves all Boolean sums $\sum_{i \in I} u_{i}$ where $\left\{u_{i}: i \in I\right\} \in C$. As usual, $j^{\prime \prime} B$ is the algebra $\{j(u): u \in B\}$; $j(B)$ is an algebra, $j(B) \supseteqq j^{\prime \prime} B$, and $j(B)$ is not necessarily complete (although $j V$-complete).

A similar observation as our Theorem 2 was used recently by $\mathrm{J}$. Silver in his result about extendable cardinals.

As a corollary of Theorem 2, we get the following theorem of Lévy and Solovay [5]: If $\kappa$ is measurable and $|B|<\kappa$, then $\kappa$ is measurable in $V^{B} .^{2}$

Let $j$ be a fixed elementary embedding of the universe. First we prove Theorem 1.

Let $M$ be a submodel.

Lemma 1. If $j_{M}$ is a class of $M$ then $M$ admits $j$.

Proof. We must show that for any formula $\varphi$,

$$
(\forall \vec{x} \in M) M \vDash(\varphi(\vec{x}) \rightarrow j M \vDash \varphi(\overrightarrow{j x})) .
$$

If $M \models \varphi(\vec{x})$, then since $M \models \varphi(\vec{x})$ is a normal formula, we have $j V \vDash$ $(j M \vDash \varphi(j(\vec{x}))$. However, $\vDash$ is absolute, so that $M \vDash(j M \vDash \varphi(j(\vec{x})))$.

Lemma 2. If $j \cap M$ is a class of $M$ and if $M$ is closed under $j$ (i.e., $j^{\prime \prime} M \subseteq M$ ), then $M$ admits $j$.

Proof. It suffices to show that $j_{M}$ is a class of $M$. Obviously, $j_{M} \cap M=j \cap M$, and because $M$ is closed under $j$, we have $j_{M} \subseteq M$, and $j_{M}=j_{M} \cap M=j \cap M$.

Now we define the model $L(j)$ :

(i ) $L_{0}(j)=0$,

(ii) $L_{\alpha}(j)=\bigcup_{\beta<\alpha} L_{\beta}(j)$ if $\alpha$ is a limit ordinal,

${ }^{2}$ An example of models which are not mild extensions but still admit $j$ are the models constructed by Kunen and Paris in [3]. 
(iii) $L_{\alpha+1}(j)=\operatorname{Def}\left(\left\langle L_{\alpha}(j), \varepsilon, j \cap L_{\alpha}(j)\right\rangle\right)$ if $\alpha$ is even,

(iv) $L_{\alpha+1}(j)=L_{\alpha}(j) \cup\left[j^{\prime \prime} L_{\alpha}(j) \cap \mathscr{P}\left(L_{\alpha}(j)\right)\right]$ if $\alpha$ is odd,

(v) $L(j)=\bigcup_{\alpha \in 0 n} L_{\alpha}(j)$.

(iii) means that $L_{\alpha+1}(j)$ consists of all subsets of $L_{\alpha}(j)$ which are definable in $L_{\alpha}(j)$ from $j \cap L_{\alpha}(j) . \mathscr{P}\left(L_{\alpha}(j)\right)$ is the set of all subset of $L_{\alpha}(j)$.

By standard methods it follows that $L_{\alpha}(j)$ is a submodel. That $L_{\alpha}(j)$ satisfies the axiom of choice is proved in Lemma 4 .

LEMMA 3. $i=j \cap L(j)$ is a class of $L(j)$ and

$$
L(j)=L(i)=L^{L(j)}(i) .
$$

Proof. By induction on $\alpha$, we prove

$$
L_{\alpha}(j)=L_{\alpha}(i)=L_{\alpha}^{L(j)}(i) .
$$

If $\alpha$ is a limit ordinal or $\alpha=\beta+1$ with $\beta$ even, then the proof is standard. Let $\beta$ be odd:

$$
\begin{aligned}
x \in L_{\beta+1}(j) & \leftrightarrow x \in L_{\beta}(j) \vee\left[x \subseteq L_{\beta}(j) \wedge x \in L(j) \wedge\left(\exists y \in L_{\beta}(j)\right)[x=j(y)]\right] \\
& \leftrightarrow x \in L_{\beta}(i) \vee\left[x \subseteq L_{\beta}(i) \wedge\left(\exists y \in L_{\beta}(i)\right)[x=i(y)]\right] \\
& \leftrightarrow x \in L_{\beta+1}(i) \\
& \leftrightarrow x \in L_{\beta+1}^{L(j)}(i) .
\end{aligned}
$$

Corollary. $L(j) \vDash V=L(i)$.

Lemma 4. $L(j) \vDash$ Axiom of Choice.

Proof. If $V=L(i)$ then there is a well ordering of the universe, definable from $i$; hence $L(j) \vDash$ Axiom of Choice.

Lemma 5. $L(j)$ is closed under $j$.

Proof. (a) If $X \subseteq O n$ and $X \in L(j)$ then there exists $\alpha$ such that $X \in L_{\alpha}(j)$ and $j(X) \leqq \alpha \subseteq L_{\alpha}(j)$; hence $j(X) \in L_{\alpha+1}(j)$ and so $j(X) \in$ $L(j)$. Similarly, if $X \subseteq O n \times O n$.

(b) If $X \in L(j)$ is arbitrary, then since $L(j) \vDash A C$, there exists a well founded relation $R \in L(j)$ on ordinals which is isomorphic to $T C(\{X\})$, the transitive closure of $\{X\}$. Hence $j(T C(\{X\}))=T C(\{j X\})$ is isomorphic to $j(R)$ which is well founded and by (a), $j R \in L(j)$; thus $j(X) \in L(j)$.

Lemma 6. If $M$ admits $j$ then 


$$
L(j)=L^{M}(j \cap M) \leqq M .
$$

Proof. Same as of Lemma 3.

Now, Theorem 1 follows.

Let $B$ be a complete Boolean algebra. The Cohen extension $V^{B}$ is the Boolean-valued model of Scott [7] or Vopènka [8]. There is a natural embedding $x \mapsto \check{x}$ of $V$ into $V^{B}$ and $C \mapsto \check{C}$ can be defined also for classes, in a natural way (in $\left(^{* * *}\right)$, we should rather write $i \supseteqq \breve{j}$ ). More generally, if $M$ is a submodel satisfying the axiom of choice and if $B \in M$ is an $M$-complete Boolean algebra then $M^{B}$ is the Cohen extension of $M$ by $B$.

Lemma 7. The condition in Theorem 2 is necessary.

Proof. Let $i$ be such that

(1) $V^{B} \vDash i$ is an elementary embedding of the universe and $i \supseteqq \grave{j}$.

Let $G$ be the canonical generic ultrafilter on $\check{B}$, i.e.,

$$
\begin{aligned}
G \in V^{(B)}, \operatorname{dom}(G) & =\{\check{u}: u \in B\}, \\
G(\check{u}) & =u \text { for all } u \in B .
\end{aligned}
$$

From (1) it follows that

$$
\begin{aligned}
& V^{B} \vDash i(G) \text { is an } i(\check{V}) \text {-complete ultrafilter on } i(\check{B}) \text {, i.e., } \\
& V^{B} \models i(G) \text { is a }(j V)^{\vee} \text {-complete ultrafilter on }(j B)^{\vee} \text {. }
\end{aligned}
$$

Let $f$ be the following function from $j(B)$ into $B$ :

$$
f(v)=\llbracket \check{v} \in i(G) \rrbracket .
$$

By (4), $f$ is a $j(V)$-complete homomorphism of $j(B)$ into $B$ and for all $u \in B, f(j u)=\llbracket(j u)^{\vee} \in i(G) \rrbracket=\llbracket i(\breve{u}) \in i(G) \rrbracket=\llbracket \check{u} \in G \rrbracket=u$. If we let $h=$ $j \circ f$ then $h$ is a $j(V)$-complete homomorphism of $j(B)$ onto $j^{\prime \prime} B$ and $h \mid j^{\prime \prime} B$ is the identity.

Lemma 8. The condition is sufficient.

Proof. Let $h$ be a $j(V)$-complete homomorphism of $j(B)$ onto $j^{\prime \prime} B$ such that $h(j u)=j u$ for all $u \in B$. We are supposed to find $i$ such that (1) holds. To simplify the considerations, assume that $G$ is some $V$-complete ultrafilter on $B$ and that $V[G]$ is the universe. (This is possible because

$$
V^{B} \vDash \check{V}[G] \text { is the universe, }
$$


where $G$ is the canonical generic ultrafilter defined in (2).)

Let $i(G)=h_{-1}\left(j^{\prime \prime} G\right)$. We have $i(G) \supseteqq j^{\prime \prime} G$, and

$i(G)$ is a $j(V)$-complete ultrafilter on $j(B)$.

Let $\pi_{G}: \quad V^{B} \rightarrow V[G]$ be the $G$-interpretation of $V^{B}$ :

$$
\begin{aligned}
& \pi_{G}(0)=0, \\
& \pi_{G}(x)=\left\{\pi_{G}(y): x(y) \in G\right\} .
\end{aligned}
$$

Since $j(B) \in j(V)$ is an $j(V)$-complete Boolean algebra, $j(V)^{j(B)}=$ $j\left(V^{B}\right)$ is the Cohen extension of $j(V)$ by $j(B)$; it follows from the definition of $i(G)$ that $i(G)$ is a $j(V)$-complete ultrafilter on $j(B)$. Let $\pi_{i G}:(j V)^{j^{B}} \rightarrow(j V)[i G]$ be the $i(G)$-interpretation of $(j V)^{j^{B}}$ and let

$$
i\left(\pi_{G} x\right)=\pi_{i G}(j x) \text {, for all } x \in V^{B} .
$$

Now we claim that $i$ is a function, $i$ is an elementary embedding of $V[G]$ into $(j V)[i G]$ and that $i \supseteqq j$. To prove that, note that for any formula $\varphi$ and for all $\vec{x} \in V^{B}$,

$$
\llbracket \varphi(\overrightarrow{j x}) \rrbracket_{j B}^{j V}=j \llbracket \varphi(\vec{x}) \rrbracket_{B}^{V}
$$

This can be proved by induction on the rank of $\vec{x}$ and on the complexity of $\varphi$. In particular, if $\pi_{G} x=\pi_{G} y$, then $\llbracket x=y \rrbracket_{B}^{V} \in G$, so that $\llbracket j x=j y \rrbracket_{j B}^{j V} \in j^{\prime \prime} G \subseteq i(G)$ and so $i\left(\pi_{G} x\right)=\pi_{i G}(j x)=\pi_{i G}(j y)=i\left(\pi_{G} y\right)$. Similarly, if $V[G] \vDash \varphi\left(\pi_{G} \vec{x}\right)$, then $(j V)[i G] \vDash \varphi\left(i\left(\pi_{G} \vec{x}\right)\right)$. If $x \in V$, then $i(x)=i\left(\pi_{G} \check{x}\right)=\pi_{i G}(j \check{x})=j(x)$.

This completes the proof of Theorem 2 .

\section{REFERENCES}

1. K. Gödel, The consistency of the continuum hypothesis..., Annals Math. Studies 3, Princeton 1940.

2. K. Kunen, Elementary embeddings and infinitary combinatorics, to appear.

3. K. Kunen and J. B. Paris, Boolean extensions and measurable cardinals, Annals of Math. Logic, 2 (1971), 359-377.

4. A. Lévy, A generalization of Gödel's notion of constructibility, J. Symbolic Logic 25 (1960), 147-155.

5. A. Lévy and R. Solovay, Measurable cardinals and the continuum hypothesis, Israel J. Math., 5 (1967), 234-248.

6. D. Scott, Measurable cardinals and constructible sets, Bull. Acad. Polon. Sci., 9 (1961).

7. D. Scott, Lectures on Boolean-valued models for set theory, notes for 1967 AMSASL U. C. L. A. Summer Institute.

8. P. Vopěnka, General theory of $\nabla$-models, Comment. Math. Univ. Carolinae, 8 (1967), 145-170. 
Received February 5, 1970. The preparation of this paper was partially supported by NSF Grant GP-22937.

State University of New York at Buffalo AND

University of California, Los ANgeles 


\title{
PACIFIC JOURNAL OF MATHEMATICS
}

\author{
EDITORS
}

\author{
H. SAMELSON \\ Stanford University \\ Stanford, California 94305

\section{R. HOBBY} \\ University of Washington \\ Seattle, Washington 98105
}

J. DUGUNDJI

Department of Mathematics

University of Southern California

Los Angeles, California 90007

RICHARD ARENS

University of California

Los Angeles, California 90024

\section{ASSOCIATE EDITORS}
E. F. BECKENBACH
B. H. NeUmanN
F. WoLF
K. YoshidA

\section{SUPPORTING INSTITUTIONS}

\author{
UNIVERSITY OF BRITISH COLUMBIA \\ CALIFORNIA INSTITUTE OF TECHNOLOGY \\ UNIVERSITY OF CALIFORNIA \\ MONTANA STATE UNIVERSITY \\ UNIVERSITY OF NEVADA \\ NEW MEXICO STATE UNIVERSITY \\ OREGON STATE UNIVERSITY \\ UNIVERSITY OF OREGON \\ OSAKA UNIVERSITY \\ UNIVERSITY OF SOUTHERN CALIFORNIA
}

\author{
STANFORD UNIVERSITY \\ UNIVERSITY OF TOKYO \\ UNIVERSITY OF UTAH \\ WASHINGTON STATE UNIVERSITY \\ UNIVERSITY OF WASHINGTON \\ $* * * *{ }^{*}$
AMERICAN MATHEMATICAL SOCIETY
CHEVRON RESEARCH CORPORATION
NAVAL WEAPONS CENTER
}

The Supporting Institutions listed above contribute to the cost of publication of this Journal, but they are not owners or publishers and have no responsibility for its content or policies.

Mathematical papers intended for publication in the Pacific Journal of Mathematics should be in typed form or offset-reproduced, (not dittoed), double spaced with large margins. Underline Greek letters in red, German in green, and script in blue. The first paragraph or two must be capable of being used separately as a synopsis of the entire paper. The editorial "we" must not be used in the synopsis, and items of the bibliography should not be cited there unless absolutely necessary, in which case they must be identified by author and Journal, rather than by item number. Manuscripts, in duplicate if possible, may be sent to any one of the four editors. Please classify according to the scheme of Math. Rev. Index to Vol. 39. All other communications to the editors should be addressed to the managing editor, Richard Arens, University of California, Los Angeles, California, 90024.

50 reprints are provided free for each article; additional copies may be obtained at cost in multiples of 50 .

The Pacific Journal of Mathematics is published monthly. Effective with Volume 16 the price per volume (3 numbers) is $\$ 8.00$; single issues, $\$ 3.00$. Special price for current issues to individual faculty members of supporting institutions and to individual members of the American Mathematical Society: $\$ 4.00$ per volume; single issues $\$ 1.50$. Back numbers are available.

Subscriptions, orders for back numbers, and changes of address should be sent to Pacific Journal of Mathematics, 103 Highland Boulevard, Berkeley, California, 94708.

PUBLISHED BY PACIFIC JOURNAL OF MATHEMATICS, A NON-PROFIT CORPORATION

Printed at Kokusai Bunken Insatsusha (International Academic Printing Co., Ltd.), 270, 3chome Totsuka-cho, Shinjuku-ku, Tokyo 160, Japan. 


\section{Pacific Journal of Mathematics}

Vol. 39, No. $2 \quad$ June, 1971

Edward Arthur Bertram, Permutations as products of conjugate infinite

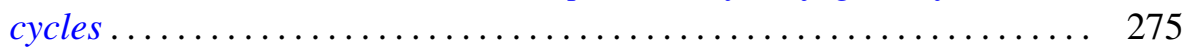

David Blair, Almost contact manifolds with Killing structure tensors ...... 285

Bruce Donald Calvert, Nonlinear equations of evolution ............. 293

Bohumil Cenkl and Giuliano Sorani, Cohomology groups associated with

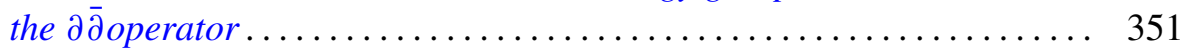

Martin Aaron Golubitsky and Bruce Lee Rothschild, Primitive subalgebras

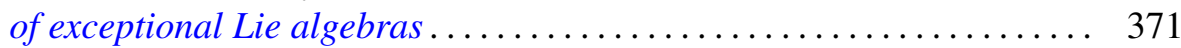

Thomas J. Jech, Two remarks on elementary embeddings of the universe ... 395

Harold H. Johnson, Conditions for isomorphism in partial differential equations........................................ 401

Solomon Leader, Measures on semilattices ..................... 407

Donald Steven Passman, Group rings satisfying a polynomial identity. II .......................................... 425

Ralph Tyrrell Rockafellar, Integrals which are convex functionals. II . . . . . 439

Stanisław Sławomir Świerczkowski, Cohomology of group germs and Lie

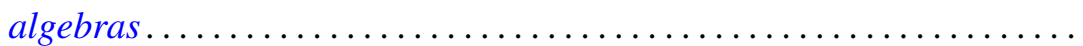

John Griggs Thompson, Nonsolvable finite groups all of whose local subgroups are solvable. III ............................ 483

Alan Curtiss Tucker, Matrix characterizations of circular-arc graphs .... 535 